\title{
Seizures during treatment of childhood acute lymphoblastic leukemia: A population-based cohort study
}

\section{Anastasopoulou, Stavroula}

2020-07

Anastasopoulou , S , Heyman, M , Eriksson, M A, Niinimäki , R, Taskinen , M , Mikkel , S , Vaitkeviciene , G E , Johannsdottir , I M , Myrberg , I H , Jonsson, O G , Als-Nielsen , B , Schmiegelow , K , Banerjee , J , Ranta , S \& Harila-Saari , A 2020 , ' Seizures during treatment of childhood acute lymphoblastic leukemia: A population-based cohort study ' , European Journal of Paediatric Neurology, vol. 27 , pp. 72-77 . https://doi.org/10.1016/j.ejpn.2020.04.004

http://hdl.handle.net/10138/329143

https://doi.org/10.1016/j.ejpn.2020.04.004

cc_by_nc_nd

acceptedVersion

Downloaded from Helda, University of Helsinki institutional repository.

This is an electronic reprint of the original article.

This reprint may differ from the original in pagination and typographic detail.

Please cite the original version. 


\section{Journal Pre-proof}

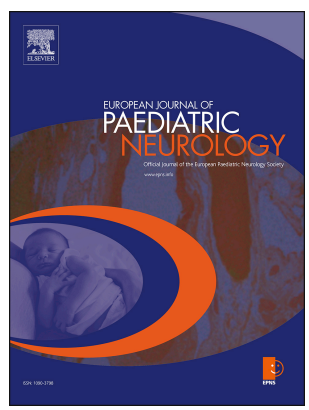

Seizures during treatment of childhood acute lymphoblastic leukemia: A populationbased cohort study

Stavroula Anastasopoulou, Mats Heyman, Mats A. Eriksson, Riitta Niinimäki, Mervi Taskinen, Sirje Mikkel, Goda E. Vaitkeviciene, Inga Maria Johannsdottir, Ida Hed Myrberg, Olafur Gisli Jonsson, Bodil Als-Nielsen, Kjeld Schmiegelow, Joanna Banerjee, Susanna Ranta, Arja Harila-Saari

PII: S1090-3798(20)30075-1

DOI: https://doi.org/10.1016/j.ejpn.2020.04.004

Reference: $\quad$ YEJPN 2663

To appear in: European Journal of Paediatric Neurology

Received Date: 22 October 2019

Revised Date: 18 March 2020

Accepted Date: 11 April 2020

Please cite this article as: Anastasopoulou S, Heyman M, Eriksson MA, Niinimäki R, Taskinen M, Mikkel S, Vaitkeviciene GE, Johannsdottir IM, Myrberg IH, Jonsson OG, Als-Nielsen B, Schmiegelow $\mathrm{K}$, Banerjee J, Ranta S, Harila-Saari A, Seizures during treatment of childhood acute lymphoblastic leukemia: A population-based cohort study, European Journal of Paediatric Neurology (2020), doi: https://doi.org/10.1016/j.ejpn.2020.04.004.

This is a PDF file of an article that has undergone enhancements after acceptance, such as the addition of a cover page and metadata, and formatting for readability, but it is not yet the definitive version of record. This version will undergo additional copyediting, typesetting and review before it is published in its final form, but we are providing this version to give early visibility of the article. Please note that, during the production process, errors may be discovered which could affect the content, and all legal disclaimers that apply to the journal pertain.

(C) 2020 Published by Elsevier Ltd on behalf of European Paediatric Neurology Society. 
Seizures during treatment of childhood acute lymphoblastic leukemia: a populationbased cohort study

Stavroula Anastasopoulou ${ }^{1,2}$, Mats Heyman ${ }^{1,2}$, Mats A. Eriksson ${ }^{1,2}$, Riitta Niinimäki ${ }^{3}$, Mervi

Taskinen $^{4}$, Sirje Mikkel $^{5}$, Goda E. Vaitkeviciene ${ }^{6}$, Inga Maria Johannsdottir ${ }^{7}$, Ida Hed

Myrberg $^{1}$, Olafur Gisli Jonsson ${ }^{8}$, Bodil Als-Nielsen ${ }^{9}$, Kjeld Schmiegelow ${ }^{9,10}$, Joanna

Banerjee $^{4}$, Susanna Ranta ${ }^{1,2^{*}}$ and Arja Harila-Saari ${ }^{11^{*}}$

*These authors contributed equally to this study.

${ }^{1}$ Karolinska Institutet, Department of Women's and Children's Health, Stockholm, Sweden

${ }^{2}$ Karolinska University Hospital, Department of Women's and Children's Health, Stockholm, Sweden

${ }^{3}$ Oulu University Hospital, Department of Children and Adolescents, and University of Oulu, PEDEGO Research Unit, Oulu, Finland.

${ }^{4}$ Division of Pediatric Hematology and Oncology and Stem Cell Transplantation, Helsinki

University Hospital and Helsinki University, Helsinki, Finland

${ }^{5}$ University of Tartu, Department of Hematology and Oncology, Tartu, Estonia

${ }^{6}$ Children's Hospital, affiliation of Vilnius University Hospital Santaros Klinikos and Vilnius University.

${ }^{7}$ Oslo University Hospital, Department of Pediatric Hematology/Oncology, Oslo, Norway

${ }^{8}$ University of Iceland, Department of Pediatrics, Reykjavík, Iceland

${ }^{9}$ Department of Paediatrics and Adolescent Medicine, Rigshospitalet, University of

Copenhagen, Copenhagen, Denmark

${ }^{10}$ Institute of Clinical Medicine, Faculty of Medicine, University of Copenhagen, Denmark 
${ }^{11}$ University of Uppsala, Department of Women's and Children's Health, Uppsala, Sweden

\section{Corresponding author:}

Stavroula Anastasopoulou, Address: Neuropediatric Unit, QB82 Astrid Lindgren's Children's Hospital, Karolinska vägen 37A, 17176 Stockholm

Email: stavroula.anastasopoulou@ki.se

Telephone: +46 (0) 851770000

Number of words in abstract: 250

Number of words in text: 2734

Number of tables: 2

Number of figures: 2

Number of supporting materials: 2

Number of references: 47

Short title: Seizures in children with acute lymphoblastic leukemia 
Abstract

Background: Seizures are common in children with acute lymphoblastic leukemia (ALL). As ALL survival rates are improving, the challenge to minimize treatment related side effects and late sequelae rises. Here, we studied the frequency, timing, etiology and risk factors of seizures in ALL patients.

Methods: The study included children aged 1-17.9 years at diagnosis of B-cell-precursor and T cell ALL who were treated according to the Nordic Society of Pediatric Hematology and Oncology (NOPHO) ALL2008 protocol between 2008 and 2015. Detailed patient data were acquired from the NOPHO ALL2008 registry and by review of medical records.

Results: Seizures occurred in 81/1464 (5.5\%) patients. The cumulative incidence of seizures at one months was $1.7 \%$ (95\% CI: 1.2-2.5) and at one year 5.3\% (95\% CI 4.2-6.5\%). Patients aged 10-17.9 years, those with T cell immunophenotype, CNS involvement, or high-risk induction with dexamethasone had higher risk for seizures in univariable analyses. Only age remained a risk factor in multivariable analyses (the cumulative incidence of seizures for patients 10-17.9 years old at one year was $9.0 \%$ (95\% CI: 6.2-12.9)). Of the 81 patients with seizures, 43 had posterior reversible encephalopathy syndrome (PRES), 15 had isolated seizures, nine had sinus venous thrombosis (SVT), three had stroke-like syndrome, and 11 had other neurotoxicities. Epilepsy diagnosis was reported in totally 11 ALL survivors at last follow up.

Conclusion: Seizures are relatively common in ALL patients and occur most often in patients with PRES, SVT, or as an isolated symptom. Older children have higher risk of seizures.

\section{Keywords: epilepsy, ALL, neurotoxicity}




\section{Abbreviations}

Abbreviation

Meaning

\begin{tabular}{|c|c|}
\hline CNS & Central Nervous System \\
\hline$A L L$ & Acute Lymphoblastic Leukemia \\
\hline PRES & Posterior Reversible Encephalopathy Syndrome \\
\hline$S V T$ & Sinus Venous Thrombosis \\
\hline$S L S$ & Stroke-Like Syndrome \\
\hline SIADH & Syndrome of Inappropriate Antidiuretic Hormone Secretion \\
\hline$B C P$ & B Cell-Precursor \\
\hline NOPHO & Nordic Society of Paediatric Haematology and Oncology \\
\hline$S C T$ & Stem Cell Transplantation \\
\hline$A D H D$ & Attention Deficit Hyperactivity Disorder \\
\hline EEG & Electroencephalogram \\
\hline$M R I$ & Magnetic Resonance Imaging \\
\hline$C T$ & Computed Tomography \\
\hline$I C U$ & Intensive Care Unit \\
\hline ILAE & International League Against Epilepsy \\
\hline$A E D$ & Antiepileptic Drug \\
\hline$N M D A$ & N-Methyl-d-Aspartate \\
\hline
\end{tabular}


1

2

3

4

\section{Introduction}

Acute neurological side effects in the central nervous system (CNS) are reported in up to $13 \%$ of children with acute lymphoblastic leukemia $(A L L)^{(1-4)}$. Seizures occur both as isolated CNS toxicity and as a symptom of systemic conditions ${ }^{(2,5,6)}$. The most frequently described neurotoxicities or systemic conditions that predispose patients to seizures are posterior reversible encephalopathy syndrome (PRES), cerebral sinus venous thrombosis (SVT), methotrexate-related stroke-like syndrome (SLS), methotrexate-related leukoencephalopathy, CNS infections, encephalopathy defined as altered mental status, and electrolyte disturbances including the syndrome of inappropriate antidiuretic hormone secretion (SIADH) ${ }^{(1,2,5-8)}$. Several chemotherapeutic agents used in ALL treatment are also associated with neurotoxicities and seizures ${ }^{(9-16)}$. Treatment of seizures is symptomatic but awareness of possible etiologies, such as PRES, infections or SVT, is important for timely diagnosis and optimal treatment ${ }^{(8,17,18)}$. The aim of this study was to explore the frequency, timing, etiology and risk factors for seizures in children with ALL as well as possible long-term effects.

\section{Materials and methods}

\subsection{Subjects and study design}

Children aged 1-17.9 years, diagnosed with B cell-precursor (BCP) or T cell ALL between July 1, 2008 and December 31, 2015 in twenty-two pediatric oncology centers in Sweden, Norway, Denmark, Finland, Iceland, Estonia, and Lithuania were included in the study. All of these centers had a common treatment protocol for childhood ALL, the Nordic Society of Paediatric Haematology and Oncology (NOPHO) ALL2008 protocol ${ }^{(19-21)}$. This protocol included an on-line toxicity registration system covering 18 toxicities (including severe neurotoxicity) with a high compliance of $95 \%^{(19)}$. Patients treated with other protocols than 
NOPHO ALL2008, such as patients diagnosed with bilineage ALL, Philadelphia positive ALL, and Down syndrome were excluded. Patients with suspected or verified neurotoxicity were retrospectively identified from the registry. Detailed data on neurological symptoms, laboratory parameters within 2 weeks prior to seizures, neuroimaging, treatment strategies, and outcome including epilepsy diagnosis at last follow-up of the patients with neurotoxicity was subsequently acquired by a questionnaire completed by the participating centers after review of medical records. NOPHO-ALL 2008 protocol provides definitions of acute CNS toxicities including seizures, PRES and methotrexate related neurotoxicity ${ }^{(19-21)}$. Further, Ponte Di Legno criteria classification of CNS toxicities in childhood ALL were applied in this study $^{(6)}$. The questionnaires were reviewed by a child neurologist (SA). Semiology of seizures was assessed by SA according to current International League Against Epilepsy (ILAE) recommendations $^{(22)}$.

\subsection{Ethics}

The NOPHO-ALL 2008 study was approved by the national Competent Authority (EudraCT 2008-003235-20 and 2011-000908-18 (Lithuania)) and the scientific Ethical Review Boards and National Medical Products Agencies in the respective countries. The families have consented to registration of ALL and treatment related toxicities for research purposes. This study is a sub-study of the ALL2008 protocol and has been approved by the NOPHO scientific board.

\subsection{Statistical analyses}

The follow-up period began with the diagnosis of ALL and continued until relapse, stem cell transplantation (SCT), secondary malignancy, death, or last follow-up date, whichever occurred first. Time to seizure was defined as days from the start of ALL treatment to the day 
of seizure, with censoring for relapse, secondary malignancy, SCT, death, other neurotoxicity, or last follow-up, whichever occurred first. Cox proportional hazards models were used for evaluating the association between possible risk factors and seizure incidence. Age group, immunophenotype, sex, CNS status at diagnosis, and risk group at diagnosis were included in the multivariable model. SPSS Version 25.0 for Windows (SPSS Inc., Chicago, IL), and R version 3.5.0, R Core Team (2019) ${ }^{(23)}$, were used for all analyses and data processing. The method of Gray ${ }^{(24)}$ was used for visualizing and calculating cumulative incidence of seizures, using the function cuminc from the R package cmprsk. Two-sided p-values below 0.05 were considered significant.

\section{Results}

\subsection{Patient population}

The study group included 1464 children; 1274 with BCP and 190 with T cell ALL. The median follow-up time for survivors was 5.0 years (range, 0.0-9.3 years, $\mathrm{n}=1351$ ) with interquartile range 3.4-6.9 years. Acute severe CNS toxicities were reported in 135/1464 patients (9.4\%). PRES, SVT, and isolated seizures were the most common neurotoxicities; seizures independently of etiology were reported in 81/135 with CNS toxicities respectively 81/1464 children with ALL.

\subsection{Incidence and risk factors for seizures}

The overall incidence of seizures under ALL treatment was 5.5\% (81/1464 patients). Two patients with seizures during ALL treatment had had previous history of febrile seizures: one patient had had generalized epilepsy but was treatment free three years prior to ALL diagnosis and one patient had been diagnosed with rolandic epilepsy with no AED treatment four years prior to ALL diagnosis. The cumulative incidence of seizures was $1.7 \%$ (95\% CI: $1.2-2.5)$ at 
one month and 5.3\% (95\% CI: 4.2-6.5) at one year from ALL diagnosis. Most seizures occurred during the first 20 weeks of treatment (induction to delayed intensification) but sporadic cases occurred throughout treatment period (Figure 1). Isolated seizures without a clearly defined cause were present in 15 cases; in 13 cases isolated seizures occurred within 3 weeks of intrathecal methotrexate administration, alone or combined with intravenous methotrexate (data missing for one patient) and they might reflect methotrexate toxicity ${ }^{(9)}$. The underlying causes of seizures in the remaining 66 patients included PRES, SVT, SLS, possible PRES (defined as a course of symptoms compatible to PRES but lacking respective neuroimaging findings), CNS infection and systemic conditions with seizures namely hyponatremia, hypoglycemia, and multiorgan failure. The clinical characteristics of all patients, with and without seizures, in this cohort are shown in Table 1. The seizures were generalized $(n=42)$, focal $(n=21)$, and focal to bilateral tonic-clonic $(n=8)$; data on semiology of seizures were not available in 10 cases (Supplementary Table 1).

\section{Older age (10-17 years compared to 1-9 years), T cell immunophenotype, CNS involvement} at diagnosis, and high-risk induction with dexamethasone treatment showed higher risk for seizures in univariable analyses (Table 1). Older age remained as risk a factor for seizures after applying multivariable analyses (Table 1 and Figure 2). The cumulative incidence of seizures in the 1-9 years group was $1.5 \%$ (95\% CI: $1.0-2.4)$ at one month and $4.3 \%$ (95\% CI: 3.2-5.6) at one year. The cumulative incidence of seizures in the 10-17.9 years group was $2.3 \%$ (95\% CI: 1.1-4.8) at one month and 9.0\% (95\% CI: 6.2-12.9) at one year.

\subsection{Other symptoms and signs in patients with seizures}

Apart from seizures, some patients also displayed signs or symptoms of encephalopathy, headache, paresis, nausea, dysphasia, dyspraxia, visual field defects, sensory disturbances, 
psychosis, fever, constipation, abdominal pain in various combinations and frequencies. See

Supplementary Figure 1 for the distribution of various symptoms among patients according to underlying CNS toxicity.

\subsection{Work-up}

Electroencephalogram (EEG) recordings were performed in 52 patients with seizures and were pathological in $43 / 51$ cases (EEG data is missing for one patient). The EEG findings, as described, include slow activity with no closer specification $(n=16)$, focal epileptiform or suspect epileptiform activity $(n=11)$, encephalopathy $(n=6)$, seizure activity including epilepsia partialis continua $(n=3)$, status epilepticus $(n=3)$, postictal status $(n=1)$, suspect generalized epileptiform activity $(n=1)$, and drug-induced abnormal activity $(n=1)$; pathological EEG result is missing for one patient.

Of the 81 patients with seizures, neuroimaging was performed at least once in 75 cases. Sixtysix patients were examined with brain magnetic resonance imaging (MRI), median days to MRI: 1 day (range: 0-20 days, data missing for 1 patient; abnormalities were observed in 58 cases). Forty-four patients were examined with brain computed tomography (CT), median days to CT: 0 days (range: 0-8 days; abnormalities were observed in 31 cases). Thirty-seven patients underwent both brain MRI and CT, median days to MRI or CT: 0 day (range: 0-16 days; abnormal findings in both examinations were present in 27 cases, only in MRI in 7 cases and only in CT in 0 cases). Neuroimaging was not performed in six patients with seizures; two of whom had isolated seizures, one had hyponatremia, one had hypoglycemia, one had possible PRES, and one had multiorgan failure. Follow up brain MRI was performed in 47 patients (data missing for three patients); median time from neurotoxicity to follow-up MRI was 5 weeks (range: 0-179 weeks; abnormalities were observed in 23 cases). Follow up 
brain CT was performed in eight patients (data missing for four patients); median time to follow up CT from neurotoxicity was 12 weeks (range: 4-52 weeks, data missing for 1 patient; abnormalities were observed in five cases).

\subsection{Treatment}

Antiepileptic drugs (AEDs) were administered to 57 patients with seizures, along with other treatments for the underlying conditions. The most frequently administered benzodiazepine was diazepam followed by midazolam, clonazepam, and clobazam. The most frequently administered AED, other than benzodiazepines, was levetiracetam followed by phenytoin, valproate, phenobarbital, topiramate, lamotrigine, and oxcarbazepine. The median duration of treatment with AEDs was 4.4 weeks (range: 0.1-177.7 weeks), data missing for 34 cases.

For the treatment of underlying neurotoxicities, 34 patients received antihypertensives, six patients received dextromethorphan (three with PRES, two with SLS and one with isolated seizures), two patients received intravenous immunoglobulins, one patient received aminophylline, and one patient received magnesium. Patients with SVT received anticoagulants and patients with bacterial or viral infections received respective treatments. Forty-two patients were admitted to the ICU during the course of their seizures and underlying causes. Modifications of chemotherapy including postponement of treatment and dose reduction were reported in 38 patients (most frequently methotrexate but also vincristine, asparaginase, mercaptopurine, high-risk block-treatment, cyclophosphamide, dexamethasone, daunorubicin, and cytarabine).

\subsection{Recurrent seizures during ALL treatment and outcome at last follow up}


At the acute phase of CNS toxicity two patients had multiple seizures lasting more than one week, seven patients had multiple seizures lasting one to seven days, 33 patients had multiple seizures lasting up to 24 hours and 24 patients had single seizures with various duration (data are missing for 15 patients).

Seven patients had repeated seizures during ALL treatment after first CNS toxicity episode occurrence (median 6 months; range 2-26 months). One of these patients had ongoing prophylactic AED medication. Recurrence of seizures was related to methotrexate treatment in two cases and recurrence of PRES in one case; no triggering factor was described in four cases. We do not have data for recurrence of seizures after the end of ALL treatment for all patients.

At the last follow-up 74/81 patients were alive, (median 5.1 years; range 1.7-9.2 years). Totally 11 ALL survivors who displayed seizures had an epilepsy diagnosis. Epilepsy diagnosis for these 11 patients was made at acute phase, reccurence of seizures or after the end of ALL-treatment (median 12 days; range 0-1535 days). Three patients had ongoing epilepsy diagnosis and AED treatment at last follow up whereas AED treatment was successfully withdrawn in eight patients after 18-40 months, median 24 months.

Nine of the 11 patients with epilepsy were initially evaluated with brain MRI. Seven patients had MRI changes typical of PRES, one patient had possible PRES with unspecific cortical ischemic lesions in the occipital lobe and in pons, and one patient had isolated seizures with discreet increased T2 signal in the right temporal lobe. A follow-up MRI was performed in eight patients and in three of them signal abnormalities remained - all previous PRES cases. Although the majority of patients with epilepsy had abnormal MRI findings and PRES as 
underlying neurotoxicity, neither pathological initial MRI findings, pathological follow-up MRI findings or PRES were statistically significant risk factors for epilepsy diagnosis (data not shown).

See Table 2 for underlying causes, work-up, treatment strategies, and outcome in patients with seizures.

\section{Discussion}

In this study, of patients with ALL treated according to the NOPHO ALL2008 protocol, 5.5\% had seizures, most commonly as a manifestation of PRES or SVT or as isolated seizures.

Children with ALL treated with various protocols have had an incidence of seizures between $1.5 \%$ and $13 \%{ }^{(1-4,25)}$. Epilepsy has previously been described as outcome of ALL patients with seizures, but the reported frequency varies presumingly due to different treatment protocols, use of neurotoxic cranial irradiation in previous studies and different length of follow up of patients ${ }^{(1,25,26)}$. Likewise, reports on recurrence of seizures under chemotherapy after first manifestation of neurotoxicity vary ${ }^{(25,26)}$. The most common underlying etiologies for seizures reported by now have been leukoencephalopathy, cerebral infarction, hypertension, or metabolic disturbances ${ }^{(25,27-29)}$. Here, PRES was the predominating underlying neurotoxicity under childhood ALL treatment in patients with seizures $\quad(5,17,30-32)$ which might reflect higher awareness of PRES and difficulties in differential diagnoses among neurotoxicities under ALL treatment ${ }^{(17,33)}$. Interestingly PRES seems to be related to epilepsy diagnosis among ALL patients, as shown even previously ${ }^{(32)}$, even though this relation did not reach statistical significance in this study. Seizures are often related to antileukemic agents $^{(3,18,25,29,34)}$. The NOPHO ALL2008 protocol $^{(19-21)}$ includes dexamethasone in high-risk induction and high doses of methotrexate, vincristine and 
asparaginase, which might contribute to the risk of neurotoxicity ${ }^{(3,5,25,34,35)}$. In contrast to an earlier study we did not find that females had a higher risk for seizures ${ }^{(25)}$.

Older age as risk factor for seizures in ALL pediatric patients was not a surprising result as older age has previously been related to a higher risk for further toxicities including thrombosis, pancreatitis, and osteonecrosis ${ }^{(36-38)}$. Adolescents with ALL have worse overall outcomes, which could be due to a higher frequency of T cell leukemia, KMT2A rearrangement in leukemic cells, and higher post-induction minimal residual disease ${ }^{(36,37)}$. Tcell leukemia, CNS involvement at diagnosis and induction with dexamethasone showed higher risk for seizures in univariate analyses but not in multivariable analyses. Another previous study on pediatric ALL and seizures showed a trend for association between more intensive ALL treatment and seizures without reaching statistical significance ${ }^{(4)}$.

The association of chemotherapeutic agents with distinct neurotoxicities should be considered in differential diagnoses. PRES was the most common underlying syndrome in patients with seizures in our study and is more frequent in the NOPHO protocols compared to other reports, possibly due to the high intensity of vincristine treatment during the first three months of treatment ${ }^{(19-21)}$. SLS is related to and occurs within three weeks of administration of methotrexate ${ }^{(39,40)}$. Most isolated seizures occurred within 3 weeks after methotrexate administration and could be attributed to methotrexate toxicity ${ }^{(9)}$. Methotrexate is administrated at least every three weeks during the first months of NOPHO ALL2008 treatment. Asparaginase and glucocorticosteroids are related to thromboses, including $\mathrm{SVT}^{(8,}$ ${ }^{41)}$. Electrolyte disturbances, hypoglycemia, CNS infections, and multiorgan failure should also be considered at the onset of seizures. MRI is the most important diagnostic examination and modern MRI techniques can be beneficial in differentiating SLS and PRES ${ }^{(42,43)}$. 
Administration of AEDs and supporting of vital bodily functions are the cornerstones for treatment of seizures. AEDs are used in the treatment of prolonged or relapsing seizures regardless of underlying etiology ${ }^{(44)}$. According to the present study, use of enzyme inducing AEDs phenytoin, phenobarbital and oxcarbazepine was common in ALL patients. However clinicians should be aware that concomitant use of liver enzyme inducers and chemotherapy should be discouraged due to diverse interactions and therefore alternative AEDs should be recommended $^{(45)}$. The decision to use prophylactic AED treatment should be grounded on the presence of risk factors for relapse, namely abnormal EEG findings and abnormal neuroimaging findings ${ }^{(46)}$. According to guidelines on withdrawal of AEDs in epilepsy, discontinuation of AEDs should be considered after 2 seizure-free years as soon as seizures are controlled with respect to clinical development and possible persistence of abnormalities in EEGs or other documented etiology ${ }^{(47)}$. There are no closer established guidelines for this patient group, but it is reasonable to consider withdrawal of AEDs earlier than 2 seizures-free years in cases where the triggering factors are removed and the patients show good recovery clinically and radiologically. Furthermore, it is important to treat the underlying cause of neurological symptoms ${ }^{(46)}$.

The retrospective nature of this study, missing data, lack of neuroimaging and EEG reviews and unclear proportion of child neurologist evaluation of seizures and details on how epilepsy diagnosis was established are limitations of our study that might have unclear impact on classification of seizures and epilepsy diagnosis. Still, the results reflect the clinical praxis and should encourage cooperation between child oncologists and child neurologists for optimal assessment of seizures and follow up of epilepsy. 
Seizures are a common adverse effect during the treatment of ALL. Older pediatric patients

250

251 have higher risk of seizures. Seizures are most often reported in patients with PRES during ALL treatment. Epilepsy diagnosis after seizures has been reported in more than every tenth ALL survivors but the frequency of long-term epilepsy has been lower.

\section{Acknowledgements}

This study was supported by grants provided by the Stockholm County Council (ALF project; SR), the Swedish Childhood Cancer Foundation, Sweden (SA; SR; MH) and the Danish Childhood Cancer Foundation (grant 2018-3713)

\section{Conflict of interest statement}

None of the authors have any conflicts of interest to disclose.

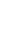




\section{References}

Baytan B, Evim MS, Guler S, Gunes AM, Okan M. Acute Central Nervous System Complications in Pediatric Acute Lymphoblastic Leukemia. Pediatr Neurol. 2015;53(4):312-8.

2. Millan NC, Pastrana A, Guitter MR, Zubizarreta PA, Monges MS, Felice MS. Acute and sub-acute neurological toxicity in children treated for acute lymphoblastic leukemia. Leuk Res. 2018;65:86-93.

3. Ochs JJ, Bowman WP, Pui CH, Abromowitch M, Mason C, Simone JV. Seizures in childhood lymphoblastic leukaemia patients. Lancet. 1984;2(8417-8418):1422-4.

4. Nassar SL, Conklin HM, Zhou Y, Ashford JM, Reddick WE, Glass JO, et al. Neurocognitive outcomes among children who experienced seizures during treatment for acute lymphoblastic leukemia. Pediatr Blood Cancer. 2017;64(8).

5. Anastasopoulou S, Eriksson MA, Heyman M, Wang C, Niinimaki R, Mikkel S, et al. Posterior reversible encephalopathy syndrome in children with acute lymphoblastic leukemia: Clinical characteristics, risk factors, course, and outcome of disease. Pediatr Blood Cancer. 2018:e27594.

6. Schmiegelow K, Attarbaschi A, Barzilai S, Escherich G, Frandsen TL, Halsey C, et al. Consensus definitions of 14 severe acute toxic effects for childhood lymphoblastic leukaemia treatment: a Delphi consensus. Lancet Oncol. 2016;17(6):e231-e9.

7. Musgrave KM, van Delft FW, Avery PJ, Clack RM, Chalmers EA, Qureshi A, et al. Cerebral sinovenous thrombosis in children and young adults with acute lymphoblastic leukaemia - a cohort study from the United Kingdom. Br J Haematol. 2017;179(4):667-9.

8. Ranta S, Tuckuviene R, Makipernaa A, Albertsen BK, Frisk T, Tedgard U, et al. Cerebral sinus venous thromboses in children with acute lymphoblastic leukaemia - a multicentre study from the Nordic Society of Paediatric Haematology and Oncology. Br J Haematol. 2015;168(4):547-52. 9. Bhojwani D, Sabin ND, Pei D, Yang JJ, Khan RB, Panetta JC, et al. Methotrexate-induced neurotoxicity and leukoencephalopathy in childhood acute lymphoblastic leukemia. J Clin Oncol. 2014;32(9):949-59.

10. Kuhlen M, Bleckmann K, Moricke A, Schrappe M, Vieth S, Escherich G, et al. Neurotoxic side effects in children with refractory or relapsed T-cell malignancies treated with nelarabine based therapy. Br J Haematol. 2017;179(2):272-83.

11. Kwong YL, Yeung DY, Chan JC. Intrathecal chemotherapy for hematologic malignancies: drugs and toxicities. Ann Hematol. 2009;88(3):193-201.

12. Magge RS, DeAngelis LM. The double-edged sword: Neurotoxicity of chemotherapy. Blood Rev. 2015;29(2):93-100.

13. Nicholson RG, Feldman W. Hyponatremia in association with vincristine therapy. Can Med Assoc J. 1972;106(4):356-7.

14. Vagace JM, de la Maya MD, Caceres-Marzal C, Gonzalez de Murillo S, Gervasini G. Central nervous system chemotoxicity during treatment of pediatric acute lymphoblastic leukemia/lymphoma. Crit Rev Oncol Hematol. 2012;84(2):274-86.

15. von Stackelberg A, Locatelli F, Zugmaier G, Handgretinger R, Trippett TM, Rizzari C, et al. Phase I/Phase II Study of Blinatumomab in Pediatric Patients With Relapsed/Refractory Acute Lymphoblastic Leukemia. J Clin Oncol. 2016;34(36):4381-9.

16. Maxwell RR, Cole PD. Pharmacogenetic Predictors of Treatment-Related Toxicity Among Children With Acute Lymphoblastic Leukemia. Curr Hematol Malig Rep. 2017;12(3):176-86. 17. Banerjee JS, Heyman M, Palomaki M, Lahteenmaki P, Arola M, Riikonen PV, et al. Posterior Reversible Encephalopathy Syndrome: Risk Factors and Impact on the Outcome in Children With Acute Lymphoblastic Leukemia Treated With Nordic Protocols. J Pediatr Hematol Oncol. 2018;40(1):e13-e8.

18. Peddi PF, Peddi S, Santos ES, Morgensztern D. Central nervous system toxicities of chemotherapeutic agents. Expert Rev Anticancer Ther. 2014;14(7):857-63. 
19.

Frandsen TL, Heyman M, Abrahamsson J, Vettenranta K, Asberg A, Vaitkeviciene G, et al. Complying with the European Clinical Trials directive while surviving the administrative pressure an alternative approach to toxicity registration in a cancer trial. Eur J Cancer. 2014;50(2):251-9. 20. Raja RA, Schmiegelow K, Albertsen BK, Prunsild K, Zeller B, Vaitkeviciene G, et al. Asparaginase-associated pancreatitis in children with acute lymphoblastic leukaemia in the NOPHO ALL2008 protocol. Br J Haematol. 2014;165(1):126-33.

21. Toft N, Birgens H, Abrahamsson J, Bernell P, Griskevicius L, Hallbook H, et al. Risk group assignment differs for children and adults 1-45 yr with acute lymphoblastic leukemia treated by the NOPHO ALL-2008 protocol. Eur J Haematol. 2013;90(5):404-12.

22. Scheffer IE, Berkovic S, Capovilla G, Connolly MB, French J, Guilhoto L, et al. ILAE classification of the epilepsies: Position paper of the ILAE Commission for Classification and Terminology. Epilepsia. 2017;58(4):512-21.

23. (2019) RCT. The R Project for Statistical Computing 2019, August 20 [Available from: https://www.r-project.org/.

24. Gray RJ. A Class of K-Sample Tests for Comparing the Cumulative Incidence of a Competing Risk. The Annals of Statistics. 1988;16(3): 1141-54.

25. Maytal J, Grossman R, Yusuf FH, Shende AC, Karayalycin G, Lanzkowsky P, et al. Prognosis and treatment of seizures in children with acute lymphoblastic leukemia. Epilepsia. 1995;36(8):831-6.

26. Khan RB, Morris EB, Pui CH, Hudson MM, Zhou Y, Cheng C, et al. Long-term outcome and risk factors for uncontrolled seizures after a first seizure in children with hematological malignancies. J Child Neurol. 2014;29(6):774-81.

27. Antunes NL. Seizures in children with systemic cancer. Pediatr Neurol. 2003;28(3):1903.

28. DiMario FJ, Jr., Packer RJ. Acute mental status changes in children with systemic cancer. Pediatrics. 1990;85(3):353-60.

29. Winick NJ, Bowman WP, Kamen BA, Roach ES, Rollins N, Jacaruso D, et al. Unexpected acute neurologic toxicity in the treatment of children with acute lymphoblastic leukemia. J Natl Cancer Inst. 1992;84(4):252-6.

30. Arzanian MT, Shamsian BS, Karimzadeh P, Kajiyazdi M, Malek F, Hammoud M. Posterior reversible encephalopathy syndrome in pediatric hematologic-oncologic disease: literature review and case presentation. Iran J Child Neurol. 2014;8(2):1-10.

31. Grioni D, Pavan F, Prunotto G, Canonico F, Grandi C, Rovelli A. Should Posterior Reversible Encephalopathy Syndrome Be Mainly Considered an Epileptic Disorder? Results of a Sequential Neurophysiological Study in a Pediatric Cohort. Neuropediatrics. 2017;48(2):72-8. 32. Khan RB, Sadighi ZS, Zabrowski J, Gajjar A, Jeha S. Imaging Patterns and Outcome of Posterior Reversible Encephalopathy Syndrome During Childhood Cancer Treatment. Pediatr Blood Cancer. 2016;63(3):523-6.

33. Hinchey J, Chaves C, Appignani B, Breen J, Pao L, Wang A, et al. A reversible posterior leukoencephalopathy syndrome. N Engl J Med. 1996;334(8):494-500.

34. Hamdan MY, Frenkel EP, Bick R. L-asparaginase-provoked seizures as singular expression of central nervous toxicity. Clin Appl Thromb Hemost. 2000;6(4):234-8.

35. Irvin W, MacDonald G, Smith JK, Kim WY. Dexamethasone-induced posterior reversible encephalopathy syndrome. J Clin Oncol. 2007;25(17):2484-6.

36. Toft N, Birgens H, Abrahamsson J, Griskevicius L, Hallbook H, Heyman M, et al. Results of NOPHO ALL2008 treatment for patients aged 1-45 years with acute lymphoblastic leukemia. Leukemia. 2018;32(3):606-15.

37. Toft N, Birgens H, Abrahamsson J, Griskevicius L, Hallbook H, Heyman M, et al. Toxicity profile and treatment delays in NOPHO ALL2008-comparing adults and children with Philadelphia chromosome-negative acute lymphoblastic leukemia. Eur J Haematol. 2016;96(2):160-9. 
401

402

403

404

405

406

407

408

409

38. Wolthers BO, Frandsen TL, Abrahamsson J, Albertsen BK, Helt LR, Heyman M, et al. Asparaginase-associated pancreatitis: a study on phenotype and genotype in the NOPHO ALL2008 protocol. Leukemia. 2017;31(2):325-32.

39. Rogers P, Pan WJ, Drachtman RA, Haines C. A Stroke Mimic: Methotrexate-induced Neurotoxicity in the Emergency Department. J Emerg Med. 2017;52(4):559-61.

40. Watanabe K, Arakawa Y, Oguma E, Uehara T, Yanagi M, Oyama C, et al. Characteristics of methotrexate-induced stroke-like neurotoxicity. Int J Hematol. 2018;108(6):630-6.

41. Ghanem KM, Dhayni RM, Al-Aridi C, Tarek N, Tamim H, Chan AKC, et al. Cerebral sinus venous thrombosis during childhood acute lymphoblastic leukemia therapy: Risk factors and management. Pediatr Blood Cancer. 2017;64(12).

42. Covarrubias DJ, Luetmer PH, Campeau NG. Posterior reversible encephalopathy syndrome: prognostic utility of quantitative diffusion-weighted MR images. AJNR Am J Neuroradiol. 2002;23(6):1038-48.

43. Haykin ME, Gorman M, van Hoff J, Fulbright RK, Baehring JM. Diffusion-weighted MRI correlates of subacute methotrexate-related neurotoxicity. J Neurooncol. 2006;76(2):153-7.

44. Glauser T, Shinnar S, Gloss D, Alldredge B, Arya R, Bainbridge J, et al. Evidence-Based Guideline: Treatment of Convulsive Status Epilepticus in Children and Adults: Report of the Guideline Committee of the American Epilepsy Society. Epilepsy Curr. 2016;16(1):48-61.

45. Relling MV, Pui CH, Sandlund JT, Rivera GK, Hancock ML, Boyett JM, et al. Adverse effect of anticonvulsants on efficacy of chemotherapy for acute lymphoblastic leukaemia. Lancet. 2000;356(9226):285-90.

46. Beghi E, De Maria G, Gobbi G, Veneselli E. Diagnosis and treatment of the first epileptic seizure: guidelines of the Italian League against Epilepsy. Epilepsia. 2006;47 Suppl 5:2-8. 47. Beghi E, Giussani G, Grosso S, ludice A, La Neve A, Pisani F, et al. Withdrawal of antiepileptic drugs: guidelines of the Italian League Against Epilepsy. Epilepsia. 2013;54 Suppl 7:2-12. 
Figure legends

414

415 Figure 1. Distribution of seizures according to underlying cause during the treatment period. PRES=posterior reversible encephalopathy syndrome, SVT=sinus venous thrombosis,

SLS=stroke like syndrome. "Other" includes possible PRES, hyponatremia, hypoglycemia, 418 central nervous system infection, and multiorgan failure.

419

420 Figure 2. Cumulative incidence of seizures.

421

422 Supplementary Figure 1. Clinical symptoms of underlying causes of seizures in pediatric

423 ALL patients. PRES=posterior reversible encephalopathy syndrome, SVT=sinus venous

424 thrombosis, SLS=stroke like syndrome. 
Table 1. Clinical characteristics of patients with seizures and risk factors for seizures.

\begin{tabular}{|c|c|c|c|c|}
\hline & Controls $(n=1383)$ & Seizure $(n=81)$ & Univariable HR (95\% CI; p) & Multivariable HR $(95 \% \mathrm{CI} ; \mathbf{p})^{* *}$ \\
\hline Age in years (median, IQR, range) & $4.5(2.8-8.3 ; 1.0-18.0)$ & $7.8(4.4-11.3 ; 1.7-17.0)$ & $1.09(1.04-1.13 ;<0.001)$ & - \\
\hline \multicolumn{5}{|l|}{ Age group, n (\%) } \\
\hline $1-9$ years & $1111(80.3)$ & $53(65.4)$ & Ref & Ref \\
\hline $10-17$ years, $\mathrm{n}(\%)$ & $272(19.7)$ & $328(34.6)$ & $2.15(1.36-3.41 ; 0.001)$ & $1.95(1.21-3.13 ; 0.01)$ \\
\hline \multicolumn{5}{|l|}{ Sex } \\
\hline Female & $632(45.7)$ & $40(49.4)$ & Ref & Ref \\
\hline Male & $751(54.3)$ & $41(50.6)$ & $0.86(0.56-1.33 ; 0.50)$ & $0.78(0.50-1.22 ; 0.28)$ \\
\hline \multicolumn{5}{|l|}{ Immunophenotype, n (\%) } \\
\hline BCP ALL & $1213(87.7)$ & $61(75.3)$ & Ref & Ref \\
\hline T cell ALL & $170(12.3)$ & $20(24.7)$ & $2.35(1.42-3.90 ;<0.001)$ & $1.60(0.64-4.05 ; 0.32)$ \\
\hline \multicolumn{5}{|l|}{ CNS status*, n (\%) } \\
\hline CNS 1 & $1205(87.1)$ & $64(79.0)$ & Ref & Ref \\
\hline CNS 2 or 3 & $174(12.6)$ & $17(21.0)$ & $1.83(1.07-3.13 ; 0.03)$ & $1.62(0.93-2.781 ; 0.09)$ \\
\hline \multicolumn{5}{|l|}{ Induction therapy, n (\%) } \\
\hline Prednisolone & $1120(81.0)$ & $56(69.1)$ & Ref & Ref \\
\hline Dexamethasone & $251(18.1)$ & $25(30.9)$ & $2.00(1.25-3.21 ;<0.001)$ & $1.09(0.45-2.65 ; 0.85)$ \\
\hline
\end{tabular}

*4 missing values for the controls ** Including Age group, sex, immunophenotype, CNS status, induction therapy. HR=hazart ratio, $\mathrm{CI}=$ confidence interval, $\mathrm{IQR}=$ interquartile range, $\mathrm{CNS}=$ central nervous system. 
Table 2. Underlying causes to seizures, work up, treatment strategies and outcome in patients with seizures.

\begin{tabular}{|c|c|}
\hline Underlying CNS toxicity & Number of patients (\%) \\
\hline PRES & $43 / 81(53.0)$ \\
\hline SVT & 9/81 (11.1) \\
\hline Isolated seizures & $15 / 81(18.5)$ \\
\hline SLS & $3 / 81(3.7)$ \\
\hline Possible PRES & $3 / 81(3.7)$ \\
\hline Hyponatremia & $3 / 81(3.7)$ \\
\hline Hypoglycemia & $2 / 81(2.5)$ \\
\hline CNS infection & $2 / 81(2.5)$ \\
\hline Multiorgan failure & $1 / 81(1.2)$ \\
\hline \multicolumn{2}{|l|}{ Neuroimaging studies } \\
\hline Brain-MRI performed & $66 / 79(83.5)$ \\
\hline Abnormal brain-MRI & $58 / 66(87.9)$ \\
\hline Brain-CT performed & $44 / 79(55.7)$ \\
\hline Abnormal brain-CT & $31 / 44(70.5)$ \\
\hline Both brain-MRI and brain-CT performed & $37 / 79(46.8)$ \\
\hline Both brain-MRI and brain-CT abnormal & $27 / 37(73.0)$ \\
\hline \multicolumn{2}{|l|}{ EEG } \\
\hline EEG performed & $52 / 77(67.5)$ \\
\hline Abnormal EEG & $43 / 51(84.3)$ \\
\hline \multicolumn{2}{|l|}{ Treatment strategies } \\
\hline Anticonvulsants/antiepileptics & $57 / 73(78.1)$ \\
\hline ICU support & $42 / 78(53.8)$ \\
\hline Temporary adjustment of chemotherapy & $38 / 78(48.7)$ \\
\hline Antihypertensives & $34 / 73(46.6)$ \\
\hline Steroids & 18/71 (25.4) \\
\hline Intravenous immunoglobulin & $2 / 73(2.7)$ \\
\hline Magnesium & $1 / 68(1.5)$ \\
\hline Aminophylline & $1 / 67(1.5)$ \\
\hline \multicolumn{2}{|l|}{ Outcome } \\
\hline Survivors & $74 / 81(91.4)$ \\
\hline Epilepsy & $11 / 70(15.7)$ \\
\hline $\begin{array}{l}\text { Denominator }<81 \text { indicates that data were not availat } \\
\text { CNS=central nervous system, PRES=posterior revers } \\
\text { thrombosis, SLS=stroke like syndrome, MRI=magne } \\
\text { EEG=electroencephalographem, ICU=intensive care }\end{array}$ & $\begin{array}{l}\text { ts regarding the respective title. } \\
\text { pathy syndrome, } \mathrm{SVT}=\text { sinus venous } \\
\text { naging, } \mathrm{CT}=\text { computed tomography, }\end{array}$ \\
\hline
\end{tabular}




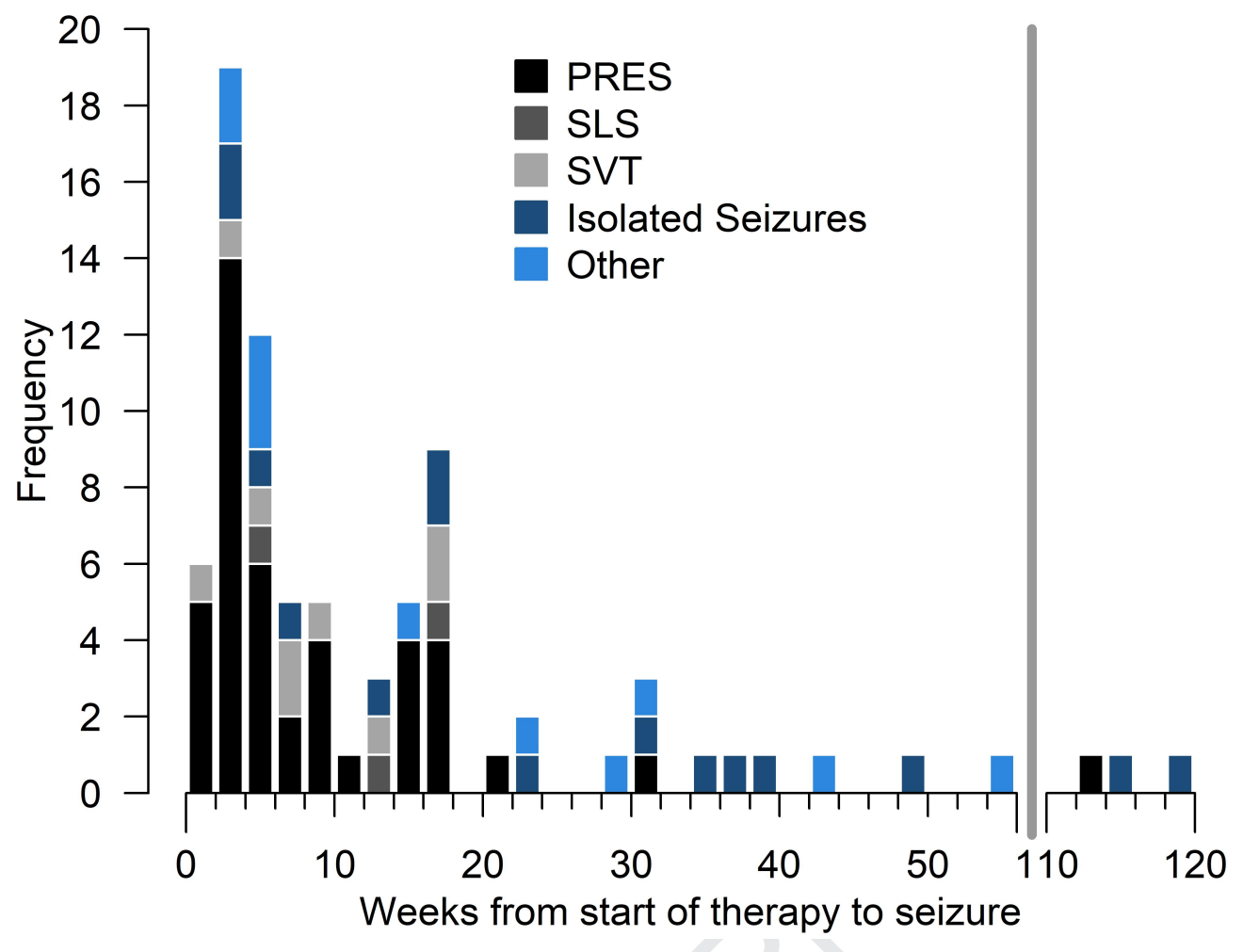



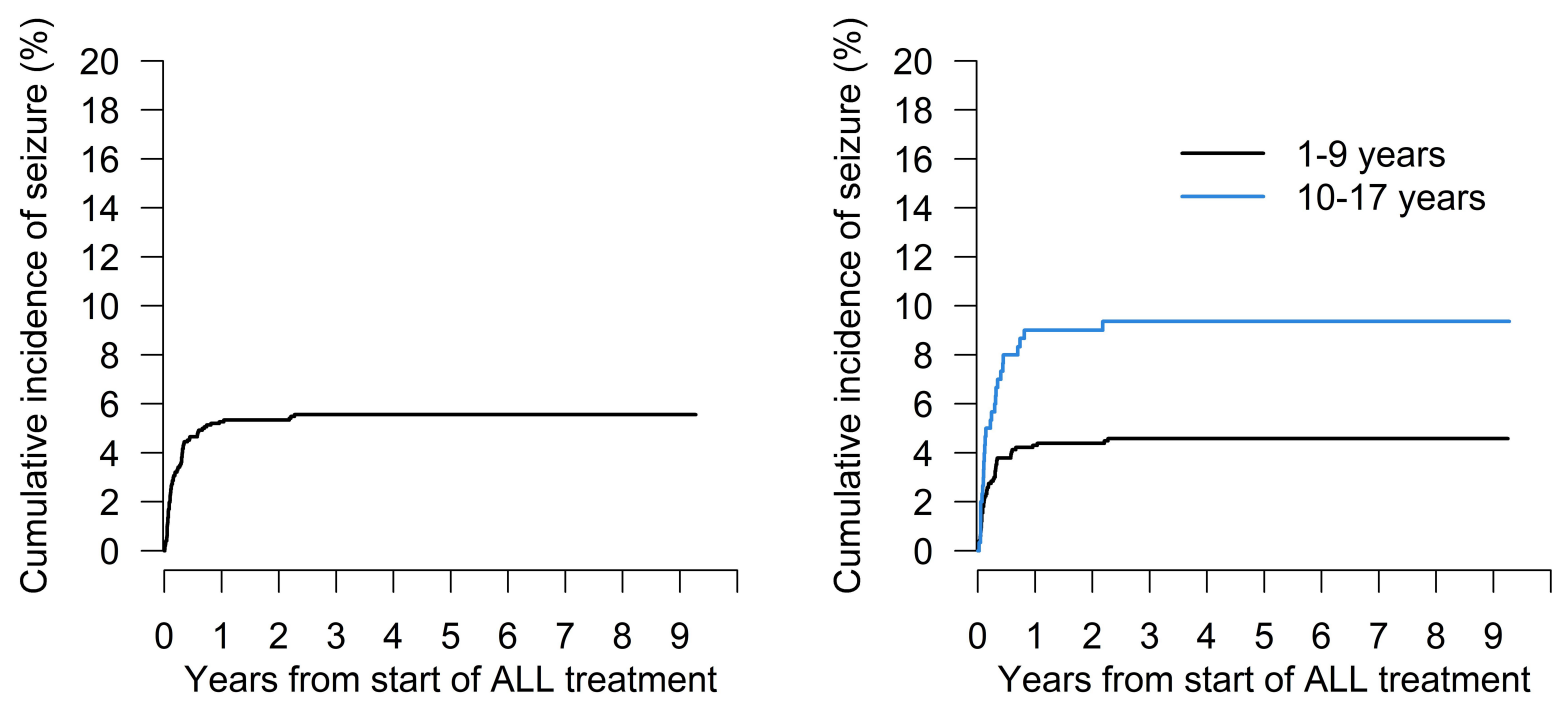


\section{Journal Pre-proof}

Seizures during treatment of childhood acute lymphoblastic leukemia: a population-

\section{based cohort study}

\section{Highlights}

Seizures are common in pediatric acute lymphoblastic leukemia (ALL) patients.

Particularly posterior reversible encephalopathy syndrome (PRES), but also sinus venous thrombosis, methotrexate related stroke-like syndrome, methotrexate, vincristine and diverse cytostatica toxicity, central nervous system infections, electrolyte disturbances were underlying causes of seizures in ALL.

Older age was an independent risk factor for seizures in pediatric ALL.

Epilepsy was common sequelae after seizures and was most common in ALL patients having displayed PRES. 
Seizures during treatment of childhood acute lymphoblastic leukemia: a populationbased cohort study

\section{Conflict of interest statement}

None of the authors have any conflicts of interest to disclose. 